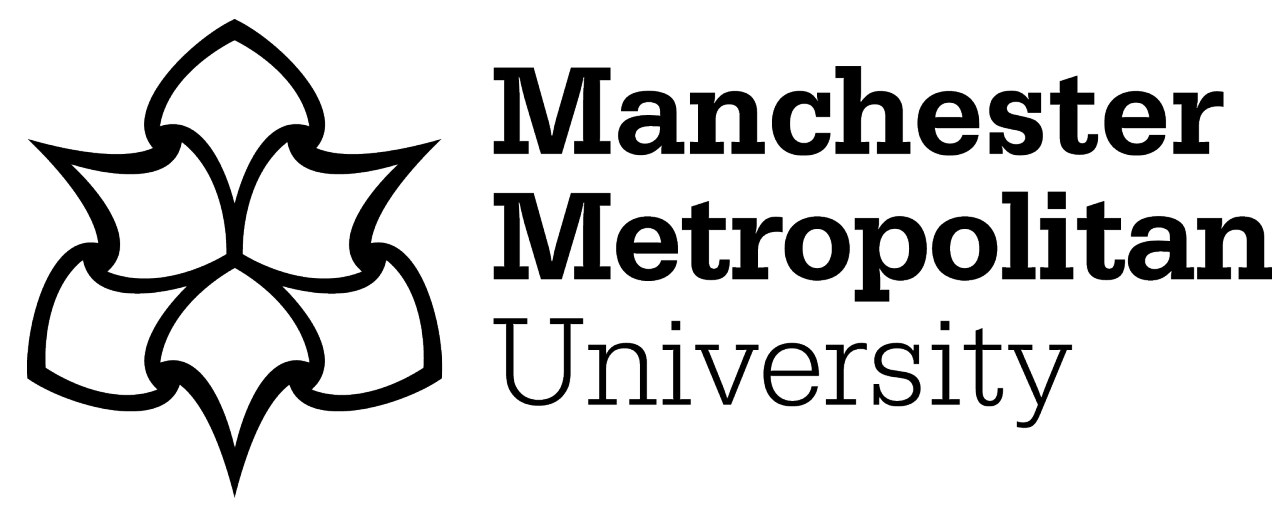

Sarwar, M, Haider, J, Persson, M and Hellbergh, H (2011) Material behavior at the extreme cutting edge in bandsawing. In: International Conference on Advances in Materials and Processing Technologies (AMPT2010), 24 October 2010 - 27 October 2010, Paris, France.

Downloaded from: https://e-space.mmu.ac.uk/622525/

DOI: https://doi.org/10.1063/1.3552320

Please cite the published version 


\title{
Material Behaviour At The Extreme Cutting Edge In Bandsawing
}

\author{
Mohammed Sarwar ${ }^{\mathrm{a}}$, Julfikar Haider ${ }^{\mathrm{a}}$, Martin Persson ${ }^{\mathrm{b}}$, and Håkan \\ Hellbergh $^{\mathrm{b}}$
}

${ }^{a}$ School of Computing Engineering and Information Sciences, Ellison Building, Northumbria University, Newcastle Upon Tyne, NE1 8ST, United Kingdom.

${ }^{b} R \& D$ Saws, SNA Europe, Fiskaregatan 1, Lidköping, Sweden.

\begin{abstract}
In recent years, bandsawing has been widely accepted as a favourite option for metal cutting off operations where the accuracy of cut, good surface finish, low kerf loss, long tool life and high material removal rate are required. Material removal by multipoint cutting tools such as bandsaw is a complex mechanism owing to the geometry of the bandsaw tooth (e.g., limited gullet size, tooth setting etc.) and the layer of material removed or undeformed chip thickness or depth of cut $(5 \mu \mathrm{m}-50 \mu \mathrm{m})$ being smaller than or equal to the cutting edge radius $(5 \mu \mathrm{m}-15$ $\mu \mathrm{m})$. This situation can lead to inefficient material removal in bandsawing. Most of the research work are concentrated on the mechanics of material removal by single point cutting tool such as lathe tool. However, such efforts are very limited in multipoint cutting tools such as in bandsaw. This paper presents the fundamental understanding of the material behaviour at the extreme cutting edge of bandsaw tooth, which would help in designing and manufacturing of blades with higher cutting performance and life. "High Speed Photography" has been carried out to analyse the material removal process at the extreme cutting edge of bandsaw tooth. Geometric model of chip formation mechanisms based on the evidences found during "High Speed Photography" and "Quick Stop" process is presented. Wear modes and mechanism in bimetal and carbide tipped bandsaw teeth are also presented.
\end{abstract}

Keywords: Bandsaw, Bandsawing, Multipoint cutting tool

PACS: 81.20.Wk

\section{INTRODUCTION}

During the last decades considerable time and effort have been devoted to understand the material behaviour around the cutting tool edge during machining through experimental investigations, analytical modeling and finite element modeling. "Quick Stop" technique and "High Speed Photography" have been employed to capture the phenomenon at the extreme cutting edge during material removal process, which enables the scientists to develop models of chip formation mechanisms. Research work are mainly concentrated on more simple machining process with single point cutting tool such as turning and complex machining process with multipoint cutting tools such as milling and drilling to some extent. The same does not apply for machining with bandsaws, which is generally considered as a low cost cutting off process. Thompson and Sarwar are among the earlier researchers [1-6], who have 
contributed to the fundamental understanding of cutting actions in bandsawing and wear in bandsaw tooth.

In recent years, the bandsaws have become popular in a variety of industries, in particular steel manufacturers, steel stockholders, forging company etc. owing to its high cutting rate, low kerf loss, good straightness of cut, competitive surface finish and long tool life [7]. However, the bandsaw designer and production engineers continue to face the demands for improving cutting efficiency (i.e., lower cost per cut), accuracy in workpiece dimensions and cut surface quality. Furthermore, the demand for efficient cutting off to size of difficult-to-cut materials such as nickel based alloys, titanium alloys and other exotic alloys pose huge challenges for the bandsaw manufacturers. In order to respond to these demands, a fundamental knowledge of the nature of the cutting process in bandsawing and wear modes and mechanisms in bandsaw tooth is essential. This paper presents a summary of the current understanding of the bandsawing process and associated wear modes and mechanisms in bandsaw tooth.

\section{CHARACTERISTICS OF BANDSAWING PROCESS}

Figure 1 illustrates the chip formation mechanisms by a lathe tool and by a bandsaw tooth. The cutting edges of bandsaw are not infinitely sharp, but possess an edge radius even though they are produced by precision grinding operation. The bandsawing process is distinctive owing to the fact that cutting edge radius is equal to or larger than the layer of material removed. This situation gives rise to inefficient cutting where material is removed by a complex combination of chip formation (continuous chips, ploughing, fragmented chips) [3, 4]. A smaller chip ratio in bandsawing (approximately 0.1) indicates that the cutting is not as efficient as cutting with nominally sharp tools at large feeds such as turning, where chip ratios can be more than 0.3. The bandsaw tooth cutting action is intermittent and the number of active cutting edges in contact with the workpiece at any instant can vary depending on the shape and size of the workpieces. Furthermore, the chip formed in bandsawing has to be accommodated within the gullets of limited size compared to unrestricted chip flow in a single point cutting operation.
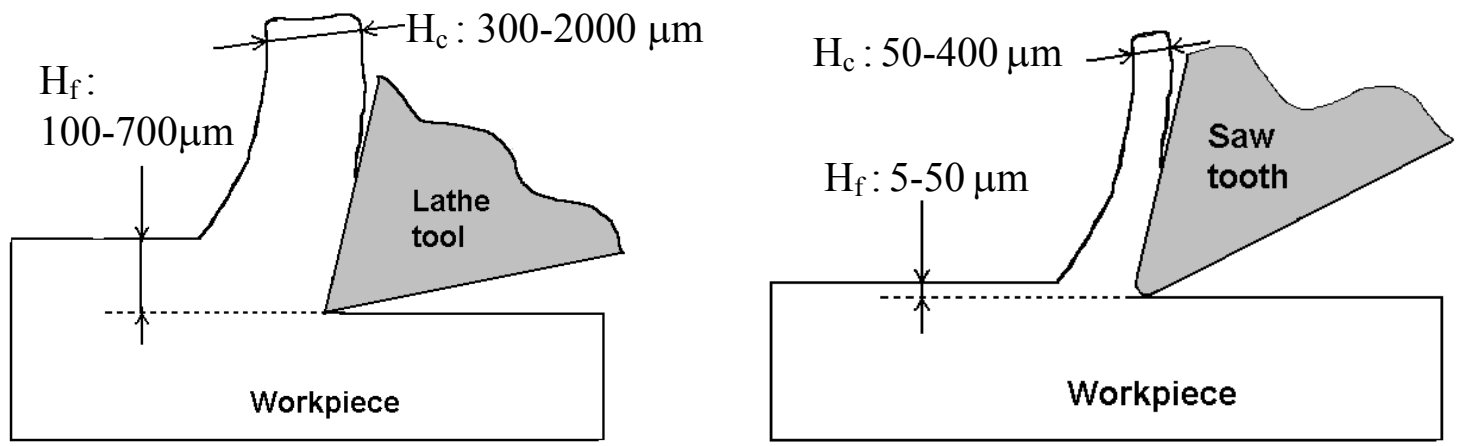

FIGURE 1. Comparisons of chip formation models in turning and bandsawing $\left(\mathrm{H}_{\mathrm{f}}\right.$ : depth of cut or undeformed chip thickness and $\mathrm{H}_{\mathrm{c}}$ : chip thickness). 


\section{MECHANICS OF CHIP FORMATION IN BANDSAWING}

\section{High Speed Photography}

High speed photography is a powerful tool to capture the dynamics of the material behaviour around the cutting edge during machining [8]. Figure 2 presents the snapshots of the sequences of chip formation process at the extreme cutting edge of a bandsaw tooth. At the beginning thin uniform chip is formed as the bandsaw tooth advances along the workpiece surface with a certain depth of cut. The workpiece material in front of the cutting edge is subsequently compressed and the chip thickness starts to increase. With further advancement of the bandsaw tooth, the workpiece material continues to build up until a saw tooth shape lump is formed. Once the lump is fully formed the same process repeats again and again. The size of the lumps varies during the chip formation process.

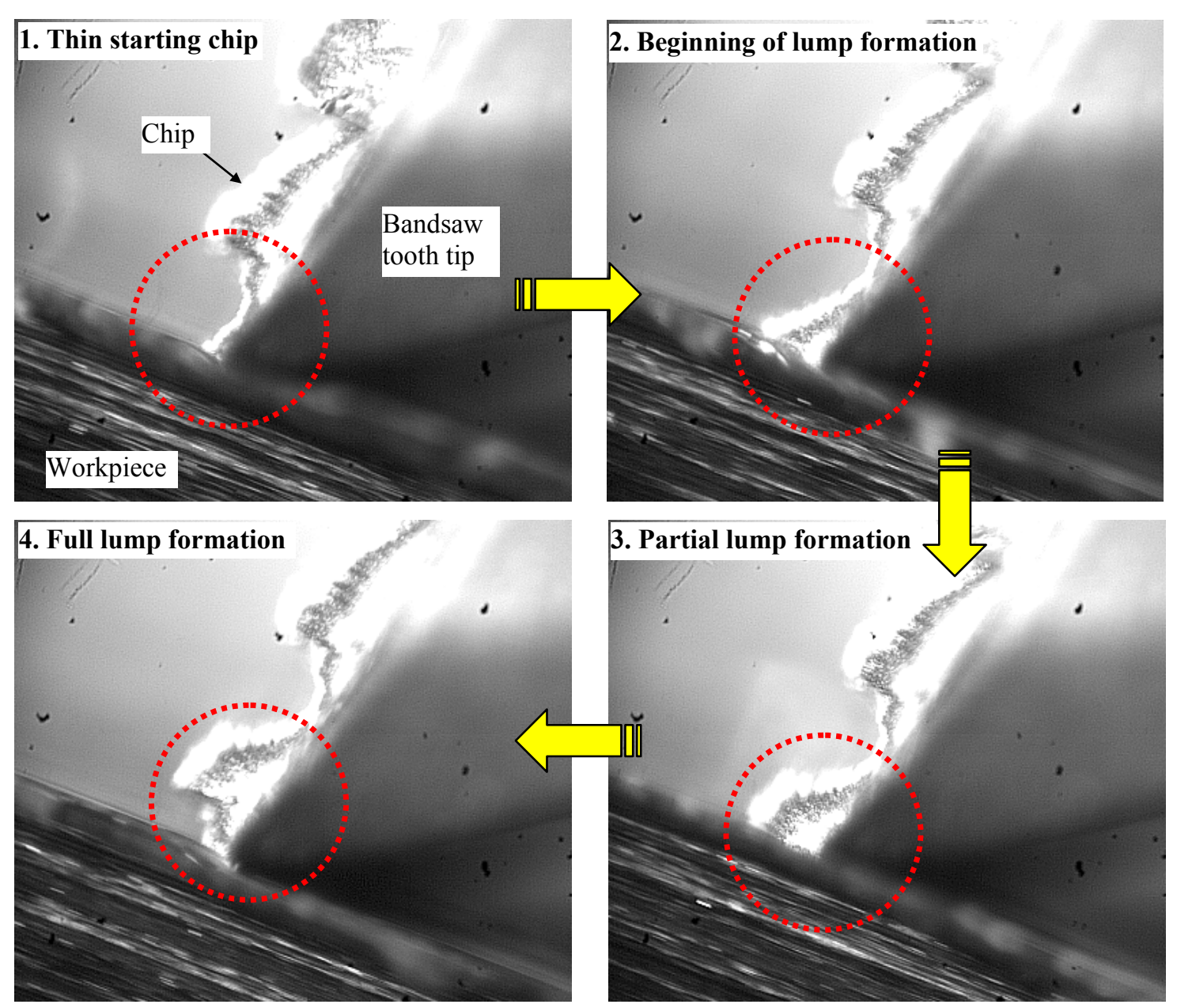

FIGURE 2. Snapshots of lumpy chip formation in bandsawing as observed in High Speed Photography (High Speed Steel bimetal bandsaw tooth machining steel workpiece). 


\section{Geometric Model}

Based on the observation of chip formation sequences in high speed photography, the schematic geometric model presented in Figure 3 can further explain the chip formation mechanisms $[3,4]$. Under the condition of cutting at smaller undeformed chip thickness with a blunt bandsaw tooth (i.e., higher edge radius), the tip of the cutting edge creates an apparent high negative rake angle with the workpiece surface. The material removal process starts with thin chip formation. With further advancement of the cutting edge, the material immediately ahead of the cutting edge becomes stagnant and forms a metal cap. This modifies the cutting edge giving metal to metal contact leading to increased friction and inefficient cutting with the chip increasing in thickness as the cut progresses. This situation continues until such time when the metal cap cannot sustain any further load and breaks down. After the breakdown of the metal cap the chip starts to flow again with a layer of material in front of the tool, extruding workpiece material to build a new metal cap. Similar to single point cutting, chips are also produced by shear type deformation during bandsawing. Chips could vary in size, shape and thickness. Continuous chips are produced when cutting soft material with bandsaw teeth having small edge radius (i.e., at the new condition of the tooth) compared to the depth of cut. Shorter and lumpy chips are formed by a combination of shearing and ploughing when the edge radius is higher than the undeformed chip thickness.

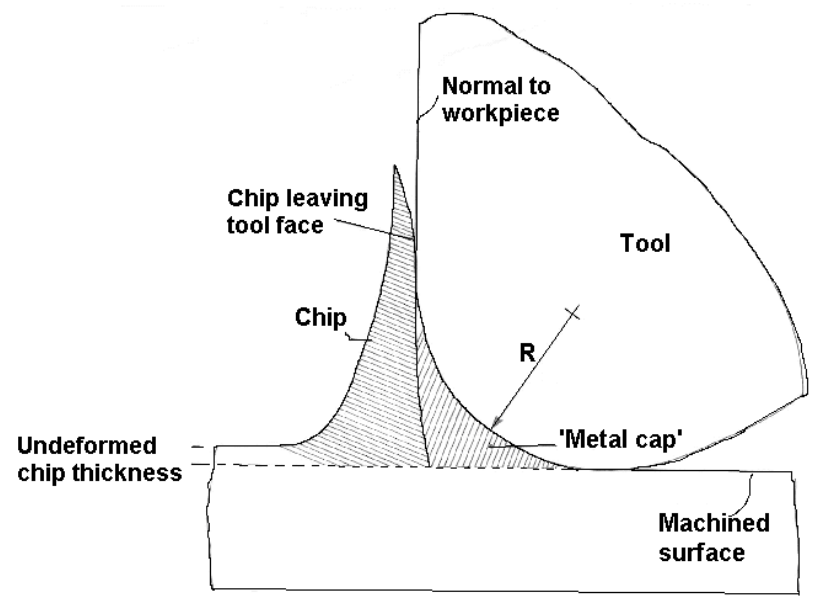

(a)

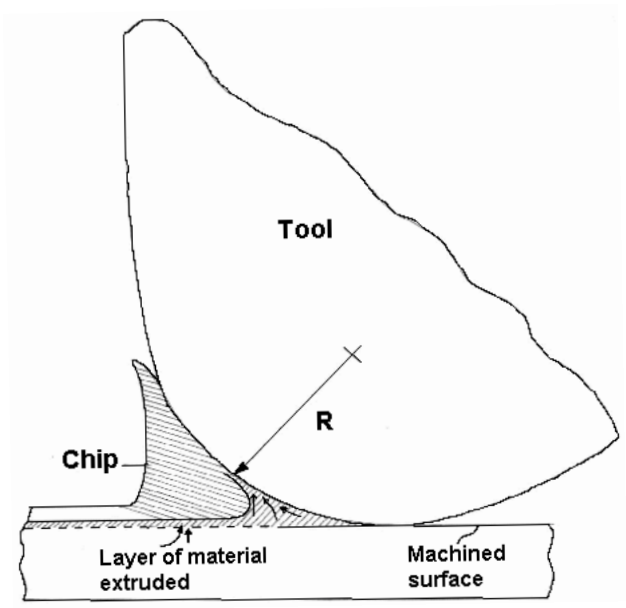

(b)

FIGURE 3. (a) Metal cap chip formation and (b) chip formation with extrusion [3].

\section{WEAR MODES AND MECHANISMS IN BANDSAW TEETH}

Bandsaw teeth are worn in such a way that wear flat is produced at the tip of each tooth and the outer corners of the set teeth are rounded as presented in Figure 4. The shape of the wear graph with the number of cuts or time is similar to the classical wear curve obtained by measuring the flank wear on a single point cutting tool showing the primary, secondary and tertiary regions. 


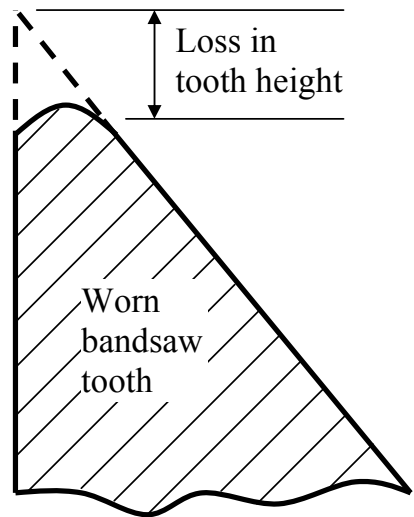

(a)

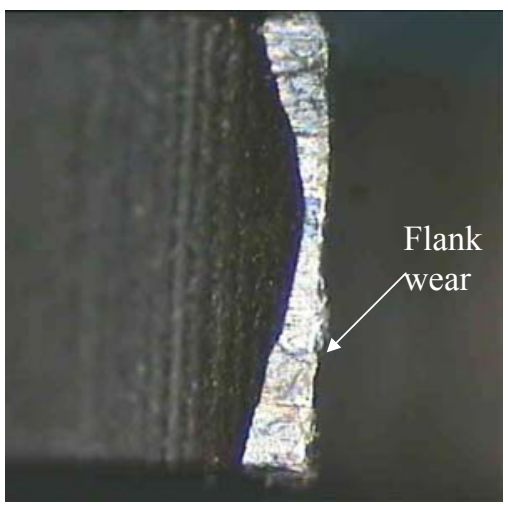

(b)

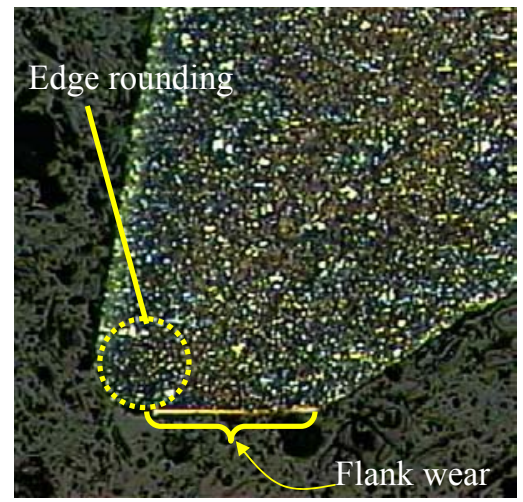

(c)

FIGURE 4. (a) Loss in bandsaw tooth height due to wear (b) top view of a worn tooth and (c) side view of a worn tooth.

In general, the principal wear modes in any bandsaw tooth can be identified as flank wear, corner wear and small rake face wear. In High Speed Steel (HSS) bimetal bandsaw tooth, flank and corner wear are developed due to the abrasive action between the tooth and the machined workpiece with small to large amount of adhesive wear depending the properties of the workpiece materials $[9,10]$. Plastic deformation of the tooth can also be observed at the end of tooth life due to the generation of excessive frictional heat. Metal cap or built-up edge is formed at the tooth edge and this could affect the cutting condition and tooth wear (Figure 5a).
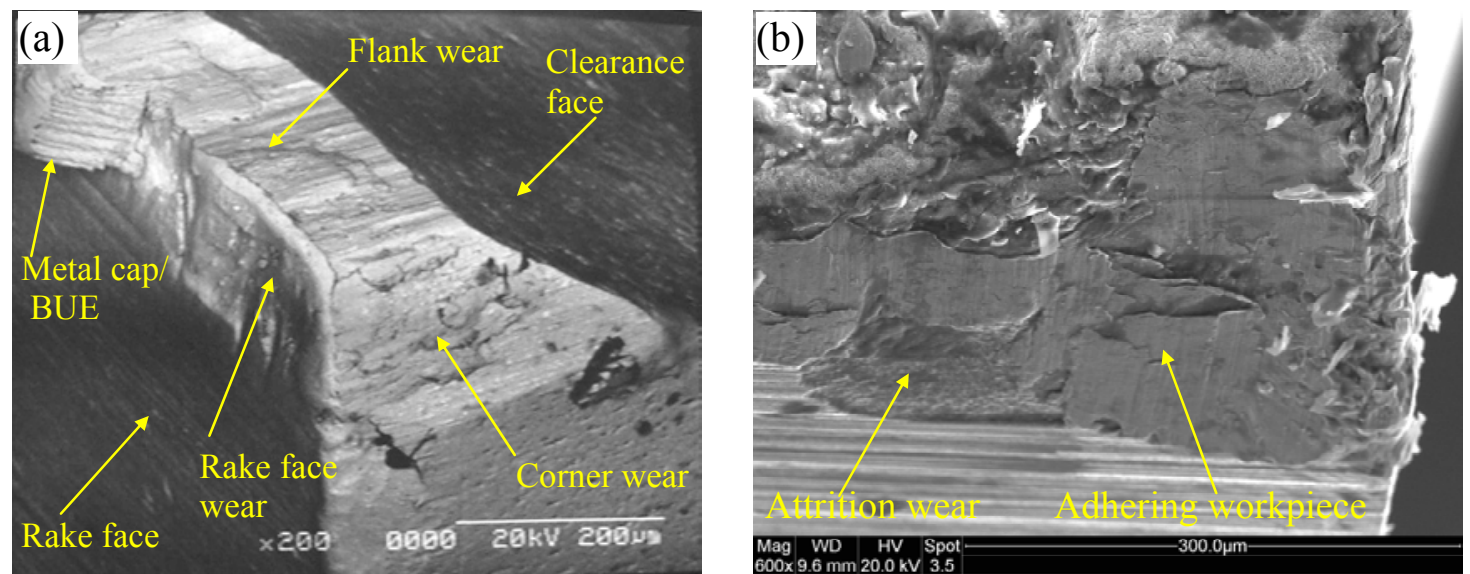

FIGURE 5. Scanning Electron Microscope pictures of worn bandsaw teeth when machining (a) stainless steel by bimetal bandsaw and (b) titanium alloy with carbide tipped bandsaw.

Whilst the basic wear modes remains the same in carbide tipped bandsaw tooth, the wear mechanisms can vary significantly. Adhesive, attrition and chipping have been identified as the governing mechanisms in carbide tooth when machining titanium alloy as presented in Figure 5(b). Furthermore, owing to the generation of high temperature at higher feeds and speeds, elements in the carbide tooth $(\mathrm{C}$ and $\mathrm{Co})$ could diffuse into the workpiece resulting tooth weakening and accelerated wear in the tooth. 
Cutting forces and specific cutting energy are found to be the important parameters to evaluate the wear condition of the bandsaw tooth through out its useful life $[10,11]$.

\section{CONCLUDING REMARKS}

High speed image processing of bandsawing operation reveals the mechanisms of chip formation during machining of steel workpiece. In bandsawing operation low depth of cut compared to the cutting edge radius leads to inefficient material removal by a combination of shearing, piling-up and ploughing processes. The chips could be either continuous or discontinuous having a distinctive characteristic of periodic lump formation. The principal wear modes in HSS steel bandsaw tooth when cutting steel workpieces can be identified as flank wear and corner wear, which are caused by abrasion and adhesion with a built-up edge formation. On the other hand chipping, adhesive wear and diffusive wear are observed in carbide tipped bandsaw tooth when machining hard-to-cut materials such as titanium alloy. The information provided concerning the material behaviour at the extreme cutting edges of bandsaw teeth would be useful for manufacturers to improve the machining performance of bandsaw by improving the tooth material, tooth design and manufacturing process.

\section{ACKNOWLEDGMENTS}

The authors would like to thank Fahd N. Khan, Research student, Northumbria University, SNA Europe and Northumbria University for supporting this research.

\section{REFERENCES}

1. P. J. Thompson, Factors influencing the sawing rate of hard ductile metals during power hacksaw and bandsaw operations, Metals Technol. 1, 437-443 (1974).

2. R. W. Taylor and P. J. Thompson, Study of bandsaw blade wear and its effects on cutting rates and economics, J. Nucl. Mater. 1, 133-140 (1976).

3. M. Sarwar and P. J. Thompson, Cutting action of blunt tools. Proceedings of International Conference on Machine Tool Design and Research, UMIST, Manchester, UK, 1981, pp. 295-303.

4. P. M. Archer, S. R. Bradbury and M. Sarwar, Evaluation of performance and wear characteristics of bandsaw blades. Proceedings of National Conference on Production Research, Huddersfield, UK, 1989, pp. 443-451.

5. M. Sarwar, D. Gillibrand and S. R. Bradbury, Forces, surface finish and friction characteristics in surface engineering single and multi-point cutting edges, Surf. Coat. Tech. 41, 443-450 (1991).

6. M. M. Ahmed, B. Hogan and E. Goode, Effect of machining parameters and workpiece shape on bandsawing process, Int. J. Mach. Tools Manuf. 29, 173-183 (1989).

7. J.V. Owen, Bandsaws join the mainstream, Manuf. Eng. 118, 28-39 (1997).

8. M. Cotterell and G. Byrne, Dynamics of chip formation during orthogonal cutting of titanium alloy Ti-6Al-4V, CIRP Annals-Manuf. Technol. 57, 93-96 (2008).

9. M. Sarwar, M. Persson and H. Hellbergh, Wear and failure modes in bandsawing operation when cutting ball bearing steels, Wear 259, 1144-1150 (2005).

10. M. Sarwar, M. Persson and H. Hellbergh, Wear of the cutting edge in the bandsawing operation when cutting austenitic 17-7 stainless steel, Wear 263, 1438-1441 (2007).

11. M. Sarwar, M. Persson, H. Hellbergh and J. Haider, Measurement of specific cutting energy for evaluating the efficiency of bandsawing different workpiece materials, Int. J. Mach. Tools Manuf. 49, 958-965 (2009). 